\title{
G11.92-0.61-MM2: A BONAFIDE MASSIVE PRESTELLAR CORE?
}

\author{
C. J. Cyganowski ${ }^{1,2}$, C. L. Brogan ${ }^{3}$, T. R. Hunter ${ }^{3}$, D. Graninger ${ }^{2}$, K. I. Öberg ${ }^{2}$,

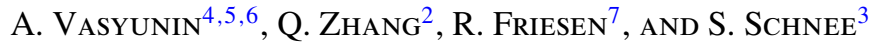 \\ ${ }^{1}$ Scottish Universities Physics Alliance (SUPA), School of Physics and Astronomy, University of St. Andrews, \\ North Haugh, St Andrews, Fife KY16 9SS, UK; cc243@st-andrews.ac.uk \\ ${ }^{2}$ Harvard-Smithsonian Center for Astrophysics, Cambridge, MA 02138, USA \\ ${ }^{3}$ NRAO, 520 Edgemont Rd, Charlottesville, VA 22903, USA \\ ${ }^{4}$ School of Physics and Astronomy, University of Leeds, Leeds LS2 9JT, UK \\ ${ }^{5}$ Max-Planck-Institut fr Extraterrestrische Physik (MPE), Giessenbachstr.1, D-85748 Garching, Germany \\ ${ }^{6}$ Ural Federal University, Ekaterinburg, Russia \\ ${ }^{7}$ Dunlap Institute for Astronomy and Astrophysics, University of Toronto, 50 St. George Street, Toronto, ON M5S 3H4, Canada \\ Received 2014 July 2; accepted 2014 October 7; published 2014 October 31
}

\begin{abstract}
Core accretion models of massive star formation require the existence of stable massive starless cores, but robust observational examples of such objects have proven elusive. We report subarcsecond-resolution Submillimeter Array (SMA) $1.3 \mathrm{~mm}, 1.1 \mathrm{~mm}$, and $0.88 \mathrm{~mm}$ and Very Large Array $1.3 \mathrm{~cm}$ observations of an excellent massive starless core candidate, G11.92-0.61-MM2, initially identified in the course of studies of GLIMPSE Extended Green Objects (EGOs). Separated by $\sim 7^{\prime \prime} .2$ from the nearby MM1 protostellar hot core, MM2 is a strong, compact dust continuum source (submillimeter spectral index $\alpha=2.6 \pm 0.1$ ), but is devoid of star formation indicators. In contrast to MM1, MM2 has no masers, no centimeter continuum, and no (sub)millimeter wavelength line emission in $\sim 24 \mathrm{GHz}$ of bandwidth observed with the SMA, including $\mathrm{N}_{2} \mathrm{H}^{+}(3-2), \mathrm{HCO}^{+}(3-2)$, and $\mathrm{HCN}(3-2)$. Additionally, there is no evidence for an outflow driven by MM2. The (sub)millimeter spectral energy distribution of MM2 is best fit with a dust temperature of $\sim 17-19 \mathrm{~K}$ and luminosity of $\sim 5-7 L_{\odot}$. The combined physical properties of MM2, as inferred from its dust continuum emission, are extreme: $M \gtrsim 30 M_{\odot}$ within a radius $<1000 \mathrm{AU}, \mathrm{N}_{\mathrm{H}_{2}}>$ $10^{25} \mathrm{~cm}^{-2}$ and $n_{\mathrm{H}_{2}}>10^{9} \mathrm{~cm}^{-3}$. Comparison of the molecular abundance limits derived from our SMA observations with gas-grain chemical models indicates that extremely dense $\left(n(\mathrm{H}) \gg 10^{8} \mathrm{~cm}^{-3}\right)$, cold $(<20 \mathrm{~K})$ conditions are required to explain the lack of observed (sub)millimeter line emission, consistent with the dust continuum results. Our data suggest that G11.92-0.61-MM2 is the best candidate for a bonafide massive prestellar core found to date, and a promising target for future higher-sensitivity observations.
\end{abstract}

Key words: astrochemistry - ISM: individual objects (G11.92-0.61) - ISM: molecules - stars: formation stars: protostars - submillimeter: ISM

Online-only material: color figures

\section{INTRODUCTION}

Do massive starless cores exist in nature? The answer to this question is a key discriminant between the two major classes of models for massive star formation: "core accretion" and "competitive accretion" (recently reviewed by Tan et al. 2014). Core accretion models require, as initial conditions, gravitationally bound, starless massive cores (e.g., McKee \& Tan 2002, 2003; Myers et al. 2013); competitive accretion models do not (e.g., Smith et al. 2009; Bonnell \& Smith 2011). In the core accretion scenario, forming a high-mass star $\left(M_{\text {ZAMS }}>\right.$ $8 M_{\odot}$ ) requires an initial core mass $\geqslant 2-3$ times larger (Alves et al. 2007; Rathborne et al. 2009; Tan et al. 2014).

Observationally, examples of massive starless cores$<0.1 \mathrm{pc}$ structures likely to form single stars or small multiple systems - have proven elusive. In addition to the candidates disqualified by sensitive mid-infrared surveys with Spitzer and Herschel, centimeter-submillimeter interferometers have revealed molecular outflows and/or masers-indisputable signs of active star formation-in past "starless" core candidates (e.g., Bontemps et al. 2010, Duarte-Cabral et al. 2013 in Cygnus-X). In this context, the best chances for identifying robust massive starless core candidates lie in massive star-forming regions for which comprehensive, high-resolution multiwavelength data sets are available.
In studying GLIMPSE Extended Green Objects (EGOs; Cyganowski et al. 2008), we have identified an excellent candidate for a massive starless core: G11.92-0.61-MM2. Our initial Submillimeter Array (SMA) $1.3 \mathrm{~mm}$ observations of the EGO G11.92-0.61 revealed a massive (proto)cluster, containing three compact cores (Cyganowski et al. 2011b, resolution $\sim 2$ '. 4). MM2 exhibited strong millimeter continuum emission, but, remarkably, no line emission across $\sim 4 \mathrm{GHz}$ of SMA bandwidth. MM2 also lacks other star formation indicators: MM2 is not associated with $\mathrm{CH}_{3} \mathrm{OH}$ maser or centimeter continuum emission, does not drive a molecular outflow, and has no $\mathrm{H}_{2} \mathrm{O}$ maser emission (Cyganowski et al. 2009, 2011a, 2011 b; Hofner \& Churchwell 1996; Breen \& Ellingsen 2011). In this Letter, we present subarcsecond-resolution $\mathrm{SMA}^{8}$ observations of G11.92-0.61 at $1.3,1.1$, and $0.88 \mathrm{~mm}$, totalling $\sim 24 \mathrm{GHz}$ of bandwidth. Together with Karl G. Jansky Very Large Array (VLA) $1.3 \mathrm{~cm} \mathrm{NH}_{3}$ and continuum observations, we use these data to constrain the physical and chemical properties of MM2, and find that MM2 is the best candidate for a bonafide massive starless core discovered to date. Throughout, we adopt the maser parallax distance of $3.37_{-0.32}^{+0.39} \mathrm{kpc}$ (Sato et al. 2014).

\footnotetext{
8 The Submillimeter Array is a joint project between the Smithsonian Astrophysical Observatory and the Academia Sinica Institute of Astronomy and Astrophysics and is funded by the Smithsonian Institution and the Academia Sinica.
} 
Table 1

Observing Parameters

\begin{tabular}{|c|c|c|c|c|}
\hline Parameter & SMA $1.3 \mathrm{~mm}$ & SMA $1.1 \mathrm{~mm}$ & SMA $0.88 \mathrm{~mm}$ & VLA $1.3 \mathrm{~cm}$ \\
\hline Observing date (UT) & 2011 Aug 28 & 2013 May 31 & 2011 Aug 19 & 2010 Aug 25, 2011 Jan 30 \\
\hline Project code & 2011A-S076 & 2013A-S043 & 2011A-S076 & $\mathrm{AB} 1346$ \\
\hline Configuration & Very Extended & Extended & Extended & $\mathrm{D}+\mathrm{CnB}$ \\
\hline$\tau_{225 \mathrm{GHz}}$ & $\sim 0.05$ & $\sim 0.1-0.15$ & $\sim 0.04-0.08$ & $\mathrm{n} / \mathrm{a}$ \\
\hline$T_{\text {sys }}($ source transit $)$ & $\sim 90 \mathrm{~K}$ & $\sim 200 \mathrm{~K}$ & $\sim 200 \mathrm{~K}$ & $\mathrm{n} / \mathrm{a}$ \\
\hline \multicolumn{5}{|l|}{ Phase Center (J2000): } \\
\hline Primary beam size (FWHP) & $52^{\prime \prime}$ & $43^{\prime \prime}$ & $34^{\prime \prime}$ & $1^{\prime} .9$ \\
\hline Frequency coverage: & & & & Mean continuum: $24.85 \mathrm{GHz}$ \\
\hline LSB & $\sim 216.9-220.9 \mathrm{GHz}$ & $\sim 265.7-269.7 \mathrm{GHz}$ & $\sim 333.6-337.6 \mathrm{GHz}$ & $8 \times 8 \mathrm{MHz}$ \\
\hline USB & $\sim 228.9-232.9 \mathrm{GHz}$ & $\sim 277.7-281.7 \mathrm{GHz}$ & $\sim 345.6-349.6 \mathrm{GHz}$ & $8 \times 8 \mathrm{MHz}$ \\
\hline Channel width ${ }^{\mathrm{a}}$ & $0.8125 \mathrm{MHz}$ & $\begin{array}{c}0.8125 \mathrm{MHz} \\
0.406 \mathrm{MHz}\left(\mathrm{N}_{2} \mathrm{H}^{+}\right)\end{array}$ & $0.8125 \mathrm{MHz}$ & $\begin{array}{l}31.25 \mathrm{kHz} \\
\left(0.4 \mathrm{~km} \mathrm{~s}^{-1}\right)\end{array}$ \\
\hline Angular resolution $^{\mathrm{c}}$ & $0^{\prime \prime} .574 \times 0^{\prime \prime} .370\left(\right.$ P.A. $\left.=30^{\circ}\right)$ & $0^{\prime \prime} 906 \times 0^{\prime \prime} 837\left(\right.$ P.A. $\left.=-74^{\circ}\right)$ & $0{ }^{\prime \prime} 798 \times 00^{\prime \prime} 703\left(\right.$ P.A. $\left.=54^{\circ}\right)$ & $1^{\prime \prime} 077 \times 0^{\prime \prime} 833\left(\right.$ P.A. $\left.=-35^{\circ}\right)$ \\
\hline Largest angular scale ${ }^{\mathrm{d}}$ & $9^{\prime \prime}$ & $9^{\prime \prime}$ & $9^{\prime \prime}$ & $\sim 40^{\prime \prime}$ \\
\hline Projected baselines & $\sim 20-394 \mathrm{k} \lambda$ & $\sim 17-191 \mathrm{k} \lambda$ & $\sim 18-225 \mathrm{k} \lambda$ & $\sim 1.8-315 \mathrm{k} \lambda$ \\
\hline Continuum rms noise $\mathrm{c}^{\mathrm{c}}$ & $0.7 \mathrm{mJy}_{\text {beam }}{ }^{-1}$ & $3 \mathrm{mJy}$ beam $^{-1}$ & $3 \mathrm{mJy}$ beam $^{-1}$ & $75 \mu \mathrm{Jy}_{\text {beam }^{-1}}$ \\
\hline Spectral line rms noise $\mathrm{e}^{\mathrm{e}}$ & $23 \mathrm{mJy}$ beam $^{-1}$ & $\begin{array}{c}71 \mathrm{mJy} \mathrm{beam}^{-1} \\
150 \mathrm{mJy} \mathrm{beam}^{-1}\left(\mathrm{~N}_{2} \mathrm{H}^{+}\right)^{\mathrm{f}}\end{array}$ & $\begin{array}{c}73 \mathrm{mJy}_{\text {beam }}^{-1} \\
55 \mathrm{mJy}^{-1} \text { beam }^{-1}\left({ }^{12} \mathrm{CO}\right)\end{array}$ & $1.5 \mathrm{mJy} \mathrm{beam}^{-1}$ \\
\hline
\end{tabular}

Notes.

a $1.1 \mathrm{~mm}$ : of 48 correlator "chunks," two have $0.406 \mathrm{MHz}$ and six have $1.625 \mathrm{MHz}$ channels.

b Using Butler-JPL-Horizons 2012 models.

${ }^{c}$ Combined LSB+USB continuum image (Briggs weighting, robust $=0.5$ ). VLA $\mathrm{NH}_{3}$ images were made with robust $=1.0$ for best sensitivity.

${ }^{\mathrm{d}}$ Scale at which $10 \%$ of peak brightness would be recovered for a Gaussian source; for 50\% recovery, multiply by 0.55 (Wilner \& Welch 1994). VLA: Estimate for combined $\mathrm{D}+\mathrm{CnB}$ images, which are dominated by the higher-weight $\mathrm{CnB}$ data.

e Typical rms per channel; Hanning-smoothed.

${ }^{\mathrm{f}}$ Near $V_{\mathrm{LSR}} \sim 35 \mathrm{~km} \mathrm{~s}^{-1}$.

\section{OBSERVATIONS}

SMA observations of G11.92-0.61 were obtained at 1.3, 1.1 , and $0.88 \mathrm{~mm}$, as summarized in Table 1 . The data were calibrated and imaged in CASA. For the 2011 data, system temperature calibration was first applied in MIRIAD; for the 2013 data, the sma2casa filler ${ }^{9}$ was used. The continuum was estimated in the $u v$-plane, using line-free channels, and subtracted from the line emission. The continuum was then selfcalibrated, and the solutions applied to the line data. For each data set, the uniform-spectral-resolution line data $(0.8125 \mathrm{MHz}$ channels) were resampled to a common velocity resolution (Table 1), then Hanning-smoothed. (To obtain better spectral resolution on the $\mathrm{N}_{2} \mathrm{H}^{+}(3-2)$ line, a mixed-spectral-resolution mode was employed, see Table 1). The ${ }^{12} \mathrm{CO}(3-2)$ data were further smoothed to $3 \mathrm{~km} \mathrm{~s}^{-1}$.

The NRAO ${ }^{10}$ VLA observations of G11.92-0.61 are part of a survey of massive protostellar objects in $1.3 \mathrm{~cm}$ continuum and line emission (Brogan et al. 2011, 2012; C. L. Brogan, in preparation; here we consider only the $\mathrm{NH}_{3}$ and $1.3 \mathrm{~cm}$ continuum data). The VLA data were calibrated, imaged, and self-calibrated in CASA.

\footnotetext{
9 http://www.cfa.harvard.edu/sma/casa/

10 The National Radio Astronomy Observatory is a facility of the National Science Foundation operated under cooperative agreement by Associated Universities, Inc.
}

Observational parameters and image properties are listed in Table 1. All measurements were made from images corrected for the primary beam response.

\section{RESULTS}

\subsection{Continuum Emission}

The continuum emission from the two brightest millimeter cores in the G11.92-0.61 protocluster, MM1 and MM2, is detected with high signal-to-noise ratio in all of our new (sub)millimeter images: observed source properties are summarized in Table 2 . Separated from MM2 by only $\sim 7^{\prime \prime} .2(0.12 \mathrm{pc})$, MM1 appears to be a typical hot core (Cyganowski et al. 2011b; Section 3.2), and so provides a useful basis for comparison. For both cores, the $\sim 0^{\prime \prime} 5$ resolution Very Extended configuration (VEX) SMA $1.3 \mathrm{~mm}$ image recovers $\sim 40 \%$ of the flux density in the $\sim 2$ ". 4 resolution $1.3 \mathrm{~mm}$ SMA image and $\sim 80 \%$ of the flux density in the $\sim 1$ ". 1 resolution CARMA $1.4 \mathrm{~mm}$ image (both from Cyganowski et al. 2011b). The fitted size of MM1 is consistently smaller than the beam (Table 2), indicating that it is unresolved in all three (sub)millimeter images. In contrast, in the highest-resolution SMA image, the fitted size of MM2 is comparable to the beam, and the source appears slightly extended (Figure 1).

To measure (sub)millimeter spectral indices, a $1.3 \mathrm{~mm}$ image was made using only those projected baselines spanned by the $1.1 \mathrm{~mm}$ data, then convolved to the $1.1 \mathrm{~mm}$ synthesized beam. 
Table 2

Properties of Continuum Sources

\begin{tabular}{|c|c|c|c|c|c|c|c|c|c|c|c|c|c|}
\hline \multirow{3}{*}{ Source } & \multicolumn{5}{|c|}{ Observed Properties } & \multirow{3}{*}{$\begin{array}{c}\text { Size } \\
(\mathrm{AU} \times \mathrm{AU})\end{array}$} & \multirow{3}{*}{$\begin{array}{c}T_{b} \\
(\mathrm{~K})\end{array}$} & \multirow{3}{*}{$\begin{array}{c}T_{\text {dust }} \\
(\mathrm{K})\end{array}$} & \multirow{3}{*}{$\tau_{\text {dust }}$} & \multirow{3}{*}{$\begin{array}{c}\kappa_{v}{ }^{\mathrm{b}} \\
\left(\mathrm{cm}^{2} \mathrm{~g}^{-1}\right)\end{array}$} & \multicolumn{3}{|c|}{ Derived Properties } \\
\hline & \multicolumn{2}{|c|}{ J2000 Coordinates ${ }^{\mathrm{a}}$} & \multirow{2}{*}{$\begin{array}{c}\text { Peak Intensity }{ }^{\mathrm{a}} \\
\left(\mathrm{mJy}^{-1}\right) \\
\left.\text { beam }^{-1}\right)\end{array}$} & \multirow{2}{*}{$\begin{array}{l}\text { Integ. Flux }{ }^{\mathrm{a}} \\
\text { Density (mJy) }\end{array}$} & \multirow{2}{*}{$\begin{array}{c}\text { Size }^{\mathrm{a}} \\
\left({ }^{\prime \prime} \times{ }^{\prime \prime}\left[\text { P.A. }^{\circ}\right]\right)\end{array}$} & & & & & & \multirow{2}{*}{$\begin{array}{l}\mathrm{M}_{\mathrm{gas}} \\
\left(M_{\odot}\right)\end{array}$} & \multirow{2}{*}{$\begin{array}{l}\mathrm{N}_{\mathrm{H}_{2}} \\
\times 10^{25} \\
\left(\mathrm{~cm}^{-2}\right)\end{array}$} & \multirow{2}{*}{$\begin{array}{c}\mathrm{n}_{\mathrm{H}_{2}} \\
\times 10^{9} \\
\left(\mathrm{~cm}^{-3}\right)\end{array}$} \\
\hline & $\alpha\left(\mathrm{hms}^{\mathrm{ms}}\right)$ & $\delta\left({ }^{\prime \prime \prime \prime}\right)$ & & & & & & & & & & & \\
\hline \multicolumn{14}{|c|}{ SMA $1.3 \mathrm{~mm}$ VEX } \\
\hline MM1 & 181358.1099 & -185420.141 & $90(1)$ & $138(2)$ & $0.34(0.01) \times 0.29(0.01)[125(7)]$ & $1150 \times 960$ & 34.0 & $150-240$ & $0.3-0.2$ & 1.11 & $3-2$ & $0.9-0.5$ & $0.9-0.5$ \\
\hline \multirow[t]{4}{*}{ MM2 } & 181357.8599 & -185413.958 & $44(1)$ & $90(2)$ & $0.56(0.02) \times 0.36(0.03)[71(5)]$ & $1880 \times 1220$ & 10.8 & 16 & 1.1 & 1.00 & 47 & 6.0 & 4.0 \\
\hline & & & & & & & & 17 & 1.0 & 1.00 & 41 & 5.3 & 3.5 \\
\hline & & & & & & & & 19 & 0.8 & 1.00 & 33 & 4.3 & 2.8 \\
\hline & & & & & & & & 20 & 0.8 & 1.00 & 30 & 3.9 & 2.6 \\
\hline \multicolumn{14}{|c|}{ SMA $1.1 \mathrm{~mm}$ EX } \\
\hline MM1 & 181358.1102 & -185420.201 & $295(5)$ & $397(6)$ & $0.66(0.02) \times 0.36(0.04)[130(2)]$ & $2210 \times 1200$ & 27.7 & $150-240$ & $0.2-0.1$ & 1.5 & $5-3$ & $0.5-0.3$ & $0.3-0.2$ \\
\hline \multirow[t]{4}{*}{ MM2 } & 181357.8568 & -185413.99 & $132(3)$ & $191(5)$ & $0.61(0.05) \times 0.55(0.05)[170(30)]$ & $2100 \times 1800$ & 9.4 & 16 & 0.9 & 1.36 & 48 & 3.8 & 1.9 \\
\hline & & & & & & & & 17 & 0.8 & 1.36 & 43 & 3.3 & 1.7 \\
\hline & & & & & & & & 19 & 0.7 & 1.36 & 35 & 2.7 & 1.4 \\
\hline & & & & & & & & 20 & 0.6 & 1.36 & 32 & 2.5 & 1.3 \\
\hline \multicolumn{14}{|c|}{ SMA $0.88 \mathrm{~mm}$ EX } \\
\hline MM1 & 181358.1066 & -185420.158 & $363(6)$ & $510(8)$ & $0.58(0.02) \times 0.34(0.03)[134(4)]$ & $1940 \times 1200$ & 27.1 & $150-240$ & $0.2-0.1$ & 2.3 & $3-2$ & $0.3-0.2$ & $0.2-0.1$ \\
\hline \multirow[t]{4}{*}{ MM2 } & 181357.8546 & -185413.92 & $167(5)$ & $294(9)$ & $0.66(0.05) \times 0.64(0.05)[4(46)]$ & $2200 \times 2200$ & 7.3 & 16 & 0.6 & 2.11 & 31 & 1.9 & 0.9 \\
\hline & & & & & & & & 17 & 0.6 & 2.11 & 27 & 1.7 & 0.8 \\
\hline & & & & & & & & 19 & 0.5 & 2.11 & 22 & 1.4 & 0.6 \\
\hline & & & & & & & & 20 & 0.5 & 2.11 & 20 & 1.3 & 0.6 \\
\hline
\end{tabular}

\begin{tabular}{|c|c|c|c|c|c|}
\hline \multicolumn{6}{|c|}{ VLA $1.3 \mathrm{~cm}$} \\
\hline CM1 & 181358.107 & -185420.20 & $0.63(0.08)$ & $<0.95$ & $<3200$ \\
\hline \multicolumn{6}{|c|}{ SMA $1.3 \mathrm{~mm}$ VEX: uvrange $<191 \mathrm{k} \lambda$, convolved to $1.1 \mathrm{~mm}$ beam } \\
\hline MM1 & 181358.1110 & -185420.144 & $121(2)$ & $148(3)$ & \\
\hline MM2 & 181357.8557 & -185413.97 & $71(2)$ & $103(3)$ & \\
\hline \multicolumn{6}{|c|}{ SMA $0.88 \mathrm{~mm} \mathrm{EX}$ : convolved to $1.1 \mathrm{~mm}$ beam } \\
\hline MM1 & 181358.1068 & -185420.160 & $388(6)$ & $525(8)$ & \\
\hline MM2 & 181357.8532 & -185413.91 & $185(6)$ & $302(9)$ & \\
\hline
\end{tabular}

Notes.

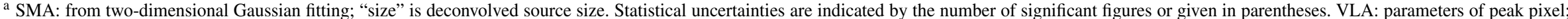
quoted uncertainties are one pixel (position) and $1 \sigma$ (peak intensity).

b Ossenkopf \& Henning (1994): grains with thin (MM1) or thick (MM2) ice mantles and coagulation at $10^{8} \mathrm{~cm}^{-3}$; linearly interpolated to $1.1 \mathrm{and} 0.88 \mathrm{~mm}$. 

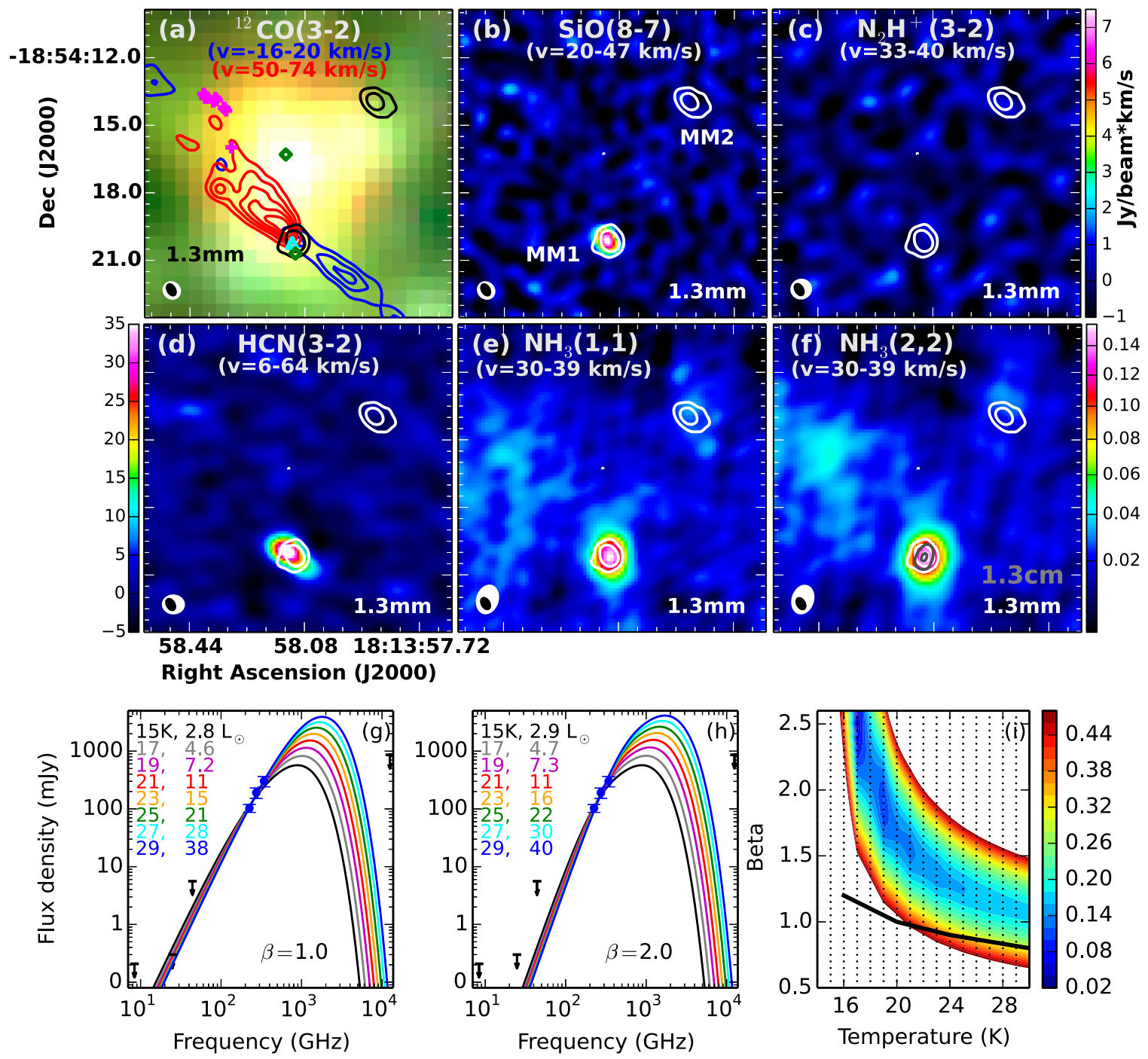

Figure 1. SMA $1.3 \mathrm{~mm}$ continuum contours overlaid on (a) three-color Spitzer image (RGB: 8.0,4.5,3.6 $\mu \mathrm{m}$ ); (b)-(f) integrated intensity maps of selected species. All panels show the same field of view; (b) and (c) and (e) and (f) share color bars. Overlaid are (a) blueshifted/redshifted ${ }^{12} \mathrm{CO}(3-2)$ and masers $(\mathrm{Class}$ II $(\diamond)$ and Class I ( + ) $\mathrm{CH}_{3} \mathrm{OH}$, Cyganowski et al. 2009; $\mathrm{H}_{2} \mathrm{O}(\triangle$ ), Hofner \& Churchwell 1996; Breen \& Ellingsen 2011) and (f) VLA $1.3 \mathrm{~cm}$ continuum contours. (g) and (h) Observed MM2 SED, overplotted with graybody fits from our $\beta$-temperature grid (Section 4.2): in the $\chi^{2}$ surface plot (i), the area below the black line is excluded by our $4 \sigma 1.3 \mathrm{~cm}$ limit. Levels: $1.3 \mathrm{~mm}:[5,25] \times \sigma, \sigma=0.7 \mathrm{mJy}_{\text {beam }}{ }^{-1} ; 1.3 \mathrm{~cm}:[5,8] \times \sigma, \sigma=75 \mu \mathrm{Jy} \mathrm{beam}^{-1} ;{ }^{12} \mathrm{CO}: 0.8 \mathrm{Jy} \mathrm{beam}^{-1} \mathrm{~km} \mathrm{~s}^{-1} \times[5,10,15](\mathrm{blue})$, $\times[5,10,15,20,25]$ (red).

(A color version of this figure is available in the online journal.)

The 0.88 and $1.1 \mathrm{~mm}$ observations have roughly comparable $u v$-coverage, so the $0.88 \mathrm{~mm}$ image was simply convolved to the $1.1 \mathrm{~mm}$ synthesized beam. Flux densities measured from these images are presented in Table 2. In fitting spectral indices, we include the statistical uncertainties (Table 2) and conservative estimates of the absolute flux calibration uncertainty $(15 \%$ at $1.3 \mathrm{~mm}, 20 \%$ at 1.1 and $0.88 \mathrm{~mm})$. The fitted spectral indices are $\alpha=3.1 \pm 0.1$ for MM1 and $\alpha=2.6 \pm 0.1$ for MM2.

Our $1.3 \mathrm{~cm}$ VLA continuum image confirms the presence of CM1 (centimeter-wavelength counterpart to MM1; Cyganowski et al. 2011a) at the $\sim 8 \sigma$ level (Figure 1, Table 2). Located $\sim 0$ '.07 (240 AU) southwest of the fitted position of MM1, the (unresolved) $1.3 \mathrm{~cm}$ emission is too strong to be due purely to dust $\left(\mathrm{S}_{\mathrm{dust}, 1.3 \mathrm{~cm}} \sim 0.2 \mathrm{mJy}\right.$, assuming $\alpha=3.1$ ). No $1.3 \mathrm{~cm}$ counterpart to MM2 is detected: the $4 \sigma$ limit is $0.30 \mathrm{mJy}$ beam $^{-1}$, corresponding to a limiting size $r \lesssim 17 \mathrm{AU}$ for any optically thick hypercompact H II region (following Cyganowski et al. 2011a).

\section{2. (Lack of) Line Emission}

The most remarkable characteristic of MM2 is its lack of (sub)millimeter-wavelength line emission: as shown in Figure 2, the image cubes are devoid of line emission at the MM2 position across $\sim 24 \mathrm{GHz}$ of bandwidth observed with the SMA. We searched the spectra at the MM2 continuum peak for $>4 \sigma$ excursions in $\geqslant 2$ adjacent channels. The only feature is at $v_{\text {observed }}=232.3365 \mathrm{GHz}$ : an unresolved $\sim 5.5 \sigma$ peak coincident with MM2 ( $>4 \sigma$ in one adjacent channel, $v_{\text {observed }}=$ $232.3374 \mathrm{GHz}$ ). Searching the splatalogue line catalog near the expected rest frequency (for $V_{\mathrm{LSR}} \sim 35-37 \mathrm{~km} \mathrm{~s}^{-1}$ ) returns primarily transitions with $E_{\text {upper }}>100 \mathrm{~K}$, inconsistent with the lack of other line emission: the only exception is an unidentified transition, U-232364 ( $\left.v_{\text {rest }}=232.364 \mathrm{GHz}\right)$. Without a plausible identification, the available evidence is insufficient to conclude that this weak, narrow feature represents real line emission associated with MM2. In marked contrast, MM1 exhibits copious line emission in molecules characteristic of hot 


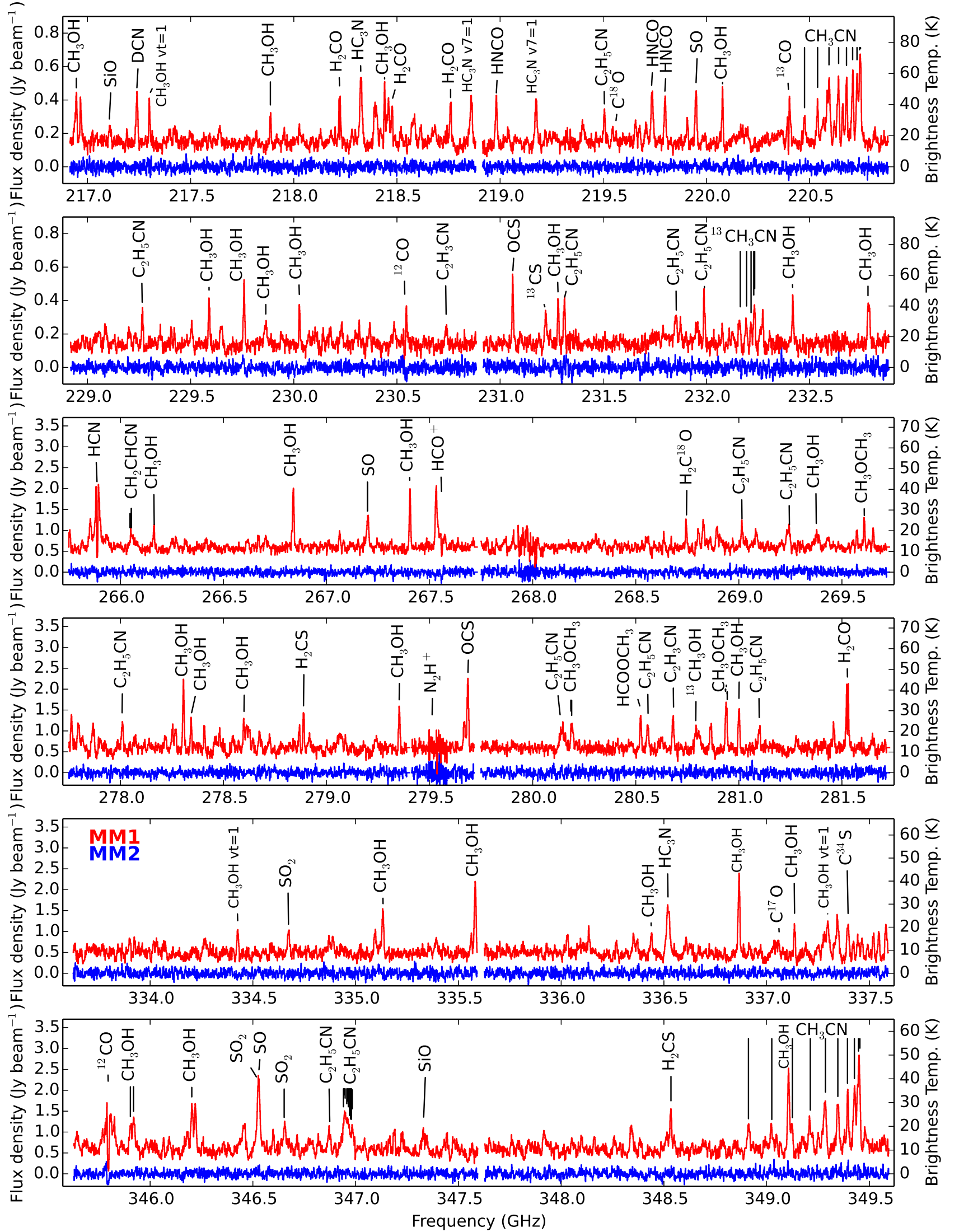

Figure 2. Continuum-subtracted SMA spectra toward the MM1 (red) and MM2 (blue) continuum peaks, showing the full 24 GHz SMA bandwidth. MM1 spectra are offset for clarity. Transition frequencies for selected molecules are labeled for reference.

(A color version of this figure is available in the online journal.) 
cores (including $\mathrm{CH}_{3} \mathrm{CN}$, OCS, $\mathrm{HC}_{3} \mathrm{~N}$ : Figure 2; Cyganowski et al. 2011b).

The SMA spectral setups (and so the nondetections toward MM2) include tracers of cold, dense gas as well as of rich hotcore chemistry. In particular, the $1.1 \mathrm{~mm}$ tuning was chosen to cover three diagnostic lines: (1) $\mathrm{N}_{2} \mathrm{H}^{+}(3-2)\left(E_{\text {upper }} \sim 27 \mathrm{~K}\right.$, $\left.n_{\text {crit }} \sim 3 \times 10^{6} \mathrm{~cm}^{-3}\right)$, for a cold $(<20 \mathrm{~K})$ core with $\mathrm{CO}$ freezeout; (2) $\mathrm{HCO}^{+}(3-2)\left(E_{\text {upper }} \sim 26 \mathrm{~K}, n_{\text {crit }} \sim 4 \times 10^{6} \mathrm{~cm}^{-3}\right)$, for a warmer core in which $\mathrm{CO}$ has come off the grains; and (3) $\operatorname{HCN}(3-2)\left(E_{\text {upper }} \sim 26 \mathrm{~K}, n_{\text {crit }} \sim 8 \times 10^{7} \mathrm{~cm}^{-3}\right)$, for a very high-density core. All of these lines are undetected toward MM2, as are (at $1.3 \mathrm{~mm}) \mathrm{N}_{2} \mathrm{D}^{+}(3-2)$, a tracer of the inner "deuteration zone" in low-mass starless cores (Caselli et al. 2002; Ceccarelli et al. 2014), ${ }^{12} \mathrm{CO}(2-1)$ and its isotopologues (Figures 1 and 2).

Our VLA $\mathrm{NH}_{3}$ data provide an independent line of evidence, and sensitivity to emission from lower-density gas and on larger spatial scales (Table 1). Like $\mathrm{N}_{2} \mathrm{H}^{+}, \mathrm{NH}_{3}$ does not deplete onto grains at densities $n_{\mathrm{H}_{2}} \lesssim 10^{6} \mathrm{~cm}^{-3}$ (Bergin \& Langer 1997); however, $n_{\text {crit }}$ for the $1.3 \mathrm{~cm} \mathrm{NH}_{3}$ inversion transitions is $>2$ orders of magnitude lower than for $\mathrm{N}_{2} \mathrm{H}^{+}(3-2)$. $\mathrm{NH}_{3}$ emission is detected in the vicinity of MM2, but does not appear to peak on the millimeter core (Figure 1, showing $v=30-39 \mathrm{~km} \mathrm{~s}^{-1}$; see also Section 4.3). In contrast, MM1 is associated with a compact $\mathrm{NH}_{3}$ core.

In addition to exhibiting much richer chemistry, MM1 differs from MM2 in driving a molecular outflow. As shown in Figure 1, MM1 drives a well-collimated bipolar molecular outflow, traced by high-velocity ${ }^{12} \mathrm{CO}(3-2)$ emission. $\mathrm{SiO}(8-7)$ and $\mathrm{SiO}(5-4)$ are also detected toward MM1, indicative of recently shocked gas, and hence an active outflow (Pineau des Forets et al. 1997; Cyganowski et al. 2012). The MM1 outflow was previously imaged, at lower resolution, in ${ }^{12} \mathrm{CO}(2-1), \mathrm{HCO}^{+}(1-0)$, and $\mathrm{SiO}(2-1)$ by Cyganowski et al. (2011b). The new, higherresolution data better resolve the outflow lobes, and clearly show that there is no evidence for an outflow driven by MM2.

\section{DISCUSSION: THE NATURE OF MM2}

\subsection{An Extragalactic Interloper?}

In the absence of (sub)millimeter line emission, we must consider the chance of MM2 being an extragalactic background source. The Mocanu et al. (2013) catalog, covering $771 \mathrm{deg}^{2}$ of the South Pole Telescope Sunyaev-Zel'dovich (SPT-SZ) survey, provides an excellent reference point. The SPT-SZ survey is multi-wavelength $(\lambda=3.2,2.0,1.4 \mathrm{~mm})$, allowing dusty sources to be distinguished from those dominated by synchrotron emission. The (sub)millimeter-wavelength spectral index and centimeter-wavelength nondetection of MM2 are inconsistent with synchrotron emission, so SPT-SZ dust-dominated sources are the relevant comparison. At 2".4 resolution, the $1.3 \mathrm{~mm}$ flux density of MM2 is $0.203 \pm 0.014 \mathrm{Jy}$ (Cyganowski et al. 2011b). Considering all dust-dominated SPT-SZ sources, the number density for $S_{1.4 \mathrm{~mm}}>0.2 \mathrm{Jy}$ is $\sim 0.002 \mathrm{deg}^{-2}$ (Mocanu et al. 2013, Table 9), corresponding to $4 \times 10^{-7}$ sources expected within the $1.3 \mathrm{~mm}$ SMA primary beam (FWHP). It is thus extremely unlikely that MM2 is a background extragalactic source, and we conclude that MM2 is a member of the G11.92-0.61 (proto)cluster.

\subsection{Physical Properties: Estimates from Dust Emission}

The combination of physical properties inferred for MM2 from its (sub)millimeter-wavelength continuum emission is extraordinary. We estimate the core gas mass from the observed integrated flux densities using a simple model of isothermal dust emission, correcting for the dust opacity (Cyganowski et al. 2011b, Equation (3)). These estimates, for a range of adopted dust temperatures, are presented in Table 2. Estimates for MM1 are included for comparison: for the hot core, the minimum and maximum adopted temperatures correspond to the two components required to fit the $J=12-11 \mathrm{CH}_{3} \mathrm{CN}$ spectrum (using the method of Hunter et al. 2014).

A strict lower limit to the physical temperature of MM2 is provided by its continuum brightness temperature in the $1.3 \mathrm{~mm}$ VEX image: $10.8 \mathrm{~K}$. To constrain the dust temperature and opacity index $(\beta)$, we fit the three (sub)millimeter flux densities (measured from the convolved images, Section 3.1) and their uncertainties with a single-temperature modified graybody for each point in a $\beta$-temperature grid $(\beta=0.5-3.05, \Delta \beta=0.05$; $T=12-35 \mathrm{~K}, \Delta T=1 \mathrm{~K})$. For each $\beta$-temperature combination, the only free parameter is $\tau_{1.3 \mathrm{~mm}}$ : the source size is fixed to 0.58 , the geometric mean of the fitted size at $1.1 \mathrm{~mm}$. Example graybody fits are shown in Figure 1, along with the $\chi^{2}$ surface plot for the $\beta$-temperature grid. Notably, our $1.3 \mathrm{~cm}$ upper limit independently excludes low- $\beta$ models (Figure 1 ), ${ }^{11}$ suggesting that MM2's moderate (sub)millimeter spectral index $(\alpha=$ $2.6 \pm 0.1)$ is due primarily to high optical depth, as opposed to e.g., large grains (Tobin et al. 2013).

As illustrated in Figure 1(i), the models that best fit MM2 span a fairly narrow temperature range, $\sim 17-19 \mathrm{~K}$. Adopting $T_{\text {dust }}=20 \mathrm{~K}$ as an approximate upper limit (see also Section 4.3), $M_{\text {gas }} \gtrsim 30 M_{\odot}$ (estimated from the $1.3 \mathrm{~mm} \mathrm{VEX}$ data; Table 2). Importantly, this mass is condensed into a radius $^{12}<1000 \mathrm{AU}$. Assuming spherical symmetry, this implies $\mathrm{N}_{\mathrm{H}_{2}}>10^{25} \mathrm{~cm}^{-2}$ and $n_{\mathrm{H}_{2}}>10^{9} \mathrm{~cm}^{-3}$. The luminosity estimate from the spectral energy distribution (SED) yields $L / M \sim 0.1-0.3(T=17-20 \mathrm{~K})$, indicative of the earliest phases in models of massive young stellar object evolution (Molinari et al. 2008).

\subsection{Astrochemical Modeling}

The dearth of line emission toward MM2 suggests extreme depletion of gas-species due to freeze-out onto grains, as seen in cold, dense low-mass prestellar cores $\left(n_{\mathrm{H}, \max } \sim 10^{8} \mathrm{~cm}^{-3}\right.$; Caselli 2011; Ceccarelli et al. 2014). To explore the possible physical properties consistent with the lack of molecular emission lines, we ran a grid of gas-grain astrochemical models using MONACO (Vasyunin et al. 2009) and the Ohio State University gas-grain reaction network (Garrod et al. 2008). We used MONACO to model the time evolution of gas-phase and grain surface chemistry under a fixed set of physical conditions. All models were run with oxygen-rich, low-metal elemental abundances (Graedel et al. 1982), a standard dustto-gas mass ratio and surface site density $(0.01$ and $1.5 \times$ $10^{15}$ sites $\mathrm{cm}^{-2}$, respectively; Semenov et al. 2003), a $10 \%$ reactive desorption efficiency (Vasyunin \& Herbst 2013) and a standard molecular cloud cosmic ray ionization $(\mathrm{CRI})$ rate $\left(\zeta_{\mathrm{CRI}}=\right.$ $1.3 \times 10^{-17} \mathrm{~s}^{-1}$, attenuated from the diffuse interstellar medium; Vasyunin et al. 2009; Padovani et al. 2009). The standard $\zeta_{\mathrm{CRI}}$ is probably too high for this source, since at the very high column densities inferred from the dust emission additional CR attenuation is expected, as discussed below. The model grid comprises

\footnotetext{
11 Upper limits are plotted, but not used in the graybody fits: $4 \sigma$ VLA limits at $0.7,3.6$, and $1.3 \mathrm{~cm}$ (Cyganowski et al. 2009, 2011a, this work), MIPSGAL $24 \mu \mathrm{m}$ limit (maximum flux density of a point source that would produce the observed intensity of the pixel coincident with MM2).

$12 r \equiv 1 / 2 \sqrt{\theta_{\text {min }} \theta_{\text {maj }}}$
} 

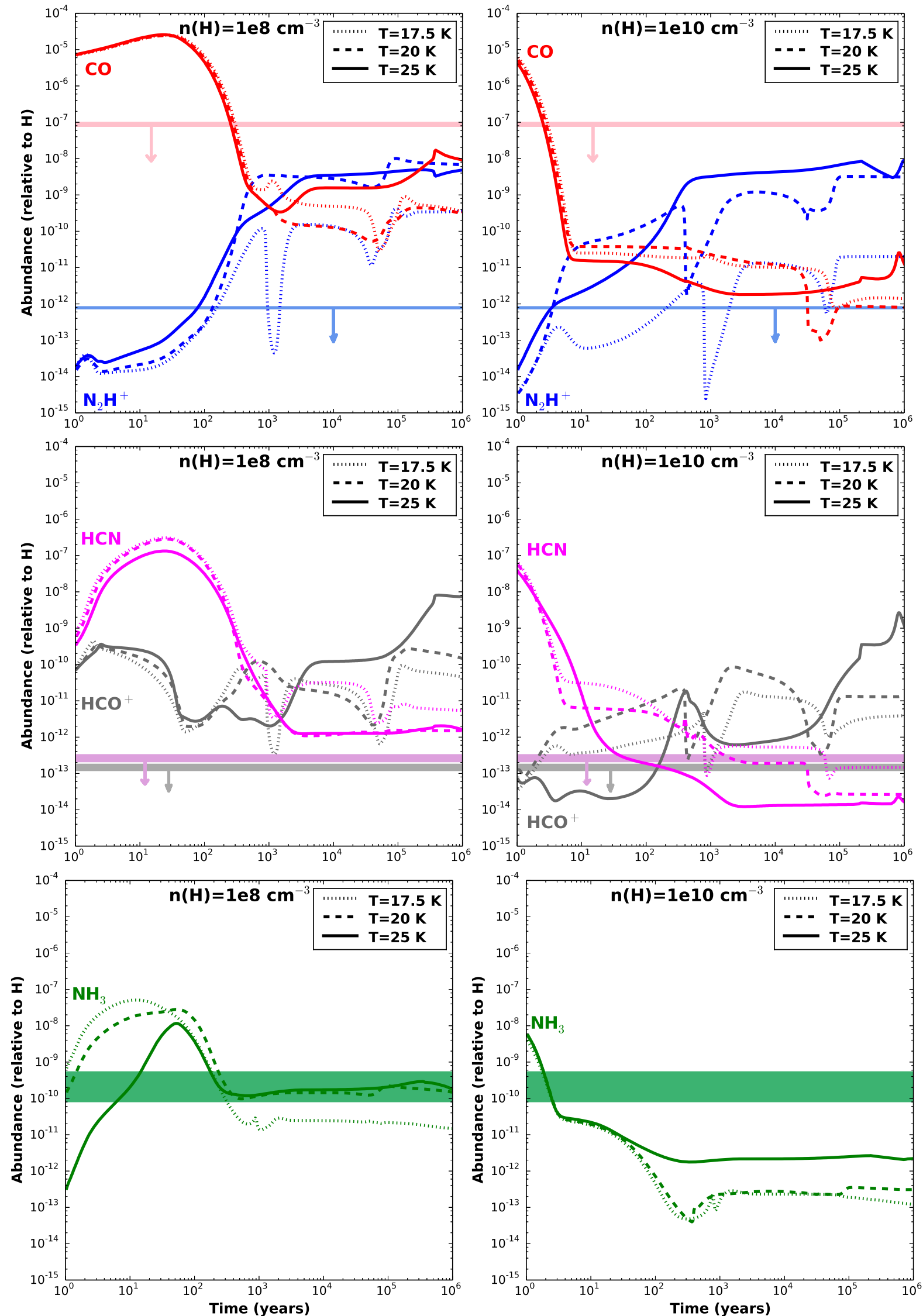

Figure 3. Time-dependent molecular abundances from gas-grain chemical models ( $A_{\mathrm{V}}=100$ ); horizontal bands show observed abundances and upper limits (with arrows). Model predictions and observations should only be compared at $t \gtrsim 10^{3}$ years, since the meaning of model predictions at shorter times is unclear. Observed $\mathrm{N}_{2} \mathrm{H}^{+}$and $\mathrm{HCN}$ limits are only reproduced at low temperatures and high densities, respectively.

(A color version of this figure is available in the online journal.) 
seven temperatures $(10-25 \mathrm{~K}, \Delta T=2.5 \mathrm{~K})$, five densities ${ }^{13}$ (1 $\left.\times 10^{8}-1 \times 10^{10} \mathrm{~cm}^{-3}\right)$, and four $A_{\mathrm{V}}(10,100,1000,10000)$. Increasing $A_{\mathrm{V}}$ above 100 has no effect; Figure 3 presents results for $A_{\mathrm{V}}=100$.

To quantitatively compare MM2's chemistry with the gasgrain models, we estimate molecular abundance limits from the SMA data for four potentially diagnostic species within our observing bands. ${ }^{14}$ Using RADEX (van der Tak et al. 2007), we find the molecular column density equivalent to our $4 \sigma T_{\mathrm{B}}$ limit for an assumed linewidth of $2 \mathrm{~km} \mathrm{~s}^{-1}$ and a suite of temperatures $(15-25 \mathrm{~K}, \Delta T=2.5 \mathrm{~K})$ informed by the SED modeling. For $\mathrm{N}_{2} \mathrm{H}^{+}$, this yields column density limits only for $T \geqslant 20 \mathrm{~K}$; if $T<20 \mathrm{~K}$ the SMA surface brightness sensitivity is insufficient to detect even optically thick emission. ${ }^{15}$ Molecular abundances, $\chi(\mathrm{MOL})=N(\mathrm{MOL}) / N(\mathrm{H})$, are calculated adopting $N(\mathrm{H})=$ $2 \times N\left(\mathrm{H}_{2}\right)$, with $N\left(\mathrm{H}_{2}\right)$ calculated from the (sub)millimeter continuum emission in the same SMA data set as the line of interest (e.g., $1.1 \mathrm{~mm}$ for $\mathrm{N}_{2} \mathrm{H}^{+}$) for each (assumed) temperature. Figure 3 presents the resulting limits as horizontal stripes: vertical extent reflects the range in assumed temperature. We emphasize that there are substantial inherent uncertainties (e.g., linewidth, temperature, estimation of $N\left(\mathrm{H}_{2}\right)$ ), and the derived abundance limits should be considered order-of-magnitude estimates. For $\mathrm{NH}_{3}$, additional uncertainty is introduced by the difference in morphology between the $\mathrm{NH}_{3}$ and dust continuum emission (Figure 1) and the difference in $u v$-coverage between the VLA and SMA data. We use the VLA spectra at the MM2 continuum peak to estimate $N\left(\mathrm{NH}_{3}\right)$ (following Cyganowski et al. 2013), and calculate $\chi\left(\mathrm{NH}_{3}\right)$ for $T_{\text {dust }}=15-25 \mathrm{~K}$ using two different measures of $N\left(\mathrm{H}_{2}\right)$ : our $1.1 \mathrm{~mm}$ SMA data and the $1.3 \mathrm{~mm}$ compact-configuration SMA data from Cyganowski et al. (2011b). Figure 3 shows the resulting $\chi\left(\mathrm{NH}_{3}\right)$ range (green rectangles).

Comparing the data and models after the initial freezeout (Figure $3 ; t \gtrsim 10^{3}$ years) shows that it is possible to explain the observed dearth of molecular emission assuming a standard gasgrain chemistry if the MM2 conditions are indeed as extreme as suggested by the SED modeling. Furthermore, specific model predictions provide three key insights. (1) The non-detection of $\mathrm{N}_{2} \mathrm{H}^{+}$rules out $T \geqslant 20 \mathrm{~K}$ for MM2. For $T \geqslant 20 \mathrm{~K}$, the model-predicted $\mathrm{N}_{2} \mathrm{H}^{+}$abundances exceed the observational limit by $\gtrsim 3$ orders of magnitude. (2) The non-detection of $\mathrm{HCN}$ favors the highest density models. For $T<20 \mathrm{~K}$ and $\mathrm{n}(\mathrm{H}) \sim 10^{10} \mathrm{~cm}^{-3}$, the model-predicted $\mathrm{HCN}$ abundances are consistent with the observational limit, within the observational uncertainties and $\sim$ order-of-magnitude uncertainty intrinsic in the model results (Vasyunin et al. 2004, 2008). Lowerdensity models $\left(\sim 10^{8} \mathrm{~cm}^{-3}\right)$ predict higher $\chi(\mathrm{HCN})$, above the observed limit for all times and temperatures. (3) The $\mathrm{NH}_{3}$ detected with the VLA near MM2 cannot trace the dense gas seen in dust continuum emission with the SMA. The inferred $\mathrm{NH}_{3}$ abundance is $\sim 10^{3}$ times higher than predicted by the cold, dense models required by the SMA nondetections of $\mathrm{N}_{2} \mathrm{H}^{+}$ and $\mathrm{HCN}$; instead, $\chi\left(\mathrm{NH}_{3}\right)$ is consistent with comparatively lower-density $\left(\sim 10^{8} \mathrm{~cm}^{-3}\right)$, warmer $(20-25 \mathrm{~K})$ material. From simple estimates, external heating by MM1/MM3 $\left(L \sim 10^{4} L_{\odot}\right.$, Cyganowski et al. 2011b) is sufficient to account for $\mathrm{NH}_{3}$ temperatures of $\sim 20 \mathrm{~K}$ near MM2.

\footnotetext{
$13 n(\mathrm{H})_{\text {total }}=n(\mathrm{H})+2 \times n\left(\mathrm{H}_{2}\right)$.

$14 \chi(\mathrm{CO})$ is estimated from $\mathrm{C}^{18} \mathrm{O}(2-1)$ assuming $N(\mathrm{CO}) / N\left(\mathrm{C}^{18} \mathrm{O}\right)=336$ (Wilson \& Rood 1994).

$15 \mathrm{~N}_{2} \mathrm{H}^{+}$is undetected toward MM1, as expected for a hot core: once CO desorbs, the $\mathrm{N}_{2} \mathrm{H}^{+}+\mathrm{CO} \rightarrow \mathrm{HCO}^{+}+\mathrm{N}_{2}$ destruction channel becomes active.
}

The poor model-data agreement for $\mathrm{HCO}^{+}$even at high densities suggests that $\zeta_{\mathrm{CRI}}$ is too high (the agreement is worse for lower-density models): a lower CRI rate (e.g., attenuated within the high-density core; Padovani et al. 2013, Figure 1) would reduce the model-predicted abundances. Overestimated desorption rates could, however, also overpredict $\chi\left(\mathrm{HCO}^{+}\right)$. Evaluating the level of $\mathrm{CR}$ attenuation would require new, deep observations of the only observable ions expected not to freeze out under any temperature and density conditions: $\mathrm{H}_{2} \mathrm{D}^{+}$and $\mathrm{D}_{2} \mathrm{H}^{+}$(e.g., Ceccarelli \& Dominik 2005).

\subsection{The Best Candidate?}

Based on the available evidence, MM2 is the best candidate for a massive starless core discovered to date. Crucially, MM2 has a centrally condensed mass sufficient to form a massive star: $\gtrsim 30 M_{\odot}$ within $\mathrm{R}<1000$ AU. Compared to CygX-N53-MM2 (another contender for "best candidate"), G11.92-0.61-MM2 has $\gtrsim 5 \times$ as much mass on $\sim 1500$ AU sizescales (estimated from millimeter continuum emission; Bontemps et al. 2010). Duarte-Cabral et al. (2013) also report a tentative outflow detection toward CygX-N53-MM2, raising the question of whether it is truly starless. Compared to the Tan et al. (2013) cores, G11.92-0.61-MM2 is more massive (with the possible exception of C1-S), more compact, and several orders of magnitude denser. G11.92-0.61-MM2 has also been more extensively and sensitively searched for star formation indicators.

Interestingly, Kauffmann et al. (2013) recently proposed that very short lifetimes could explain the lack of observational examples of massive starless cores, consistent with the very short free-fall time ( $\lesssim 1000$ yr) implied by MM2's high density. Further comparison of MM2's properties with the predictions of core accretion models for massive star formation requires additional observations, e.g., of the dense-core tracers $\mathrm{H}_{2} \mathrm{D}^{+}$, $\mathrm{N}_{2} \mathrm{H}^{+}$, and $\mathrm{N}_{2} \mathrm{D}^{+}$with the sensitivity of ALMA. Most important is detecting line emission from the dense, cold millimeter core to determine whether MM2 is gravitationally bound and will collapse to form a massive star.

Supported by NSF AAPF (C.J.C., AST-1003134) and ERC (A.V., PALs 320620). C.J.C. thanks E. Rosolowsky, K. Rowlands, and A.-M. Weijmans.

\section{REFERENCES}

Alves, J., Lombardi, M., \& Lada, C. J. 2007, A\&A, 462, L17

Bergin, E. A., \& Langer, W. D. 1997, ApJ, 486, 316

Bonnell, I. A., \& Smith, R. J. 2011, in IAU Symp. 270, Computational Star Formation, ed. J. Alves, B. Elmegreen, J. M. Girart, \& V. Trimble (Cambridge: Cambridge Univ. Press), 57

Bontemps, S., Motte, F., Csengeri, T., \& Schneider, N. 2010, A\&A, 524, A18

Breen, S. L., \& Ellingsen, S. P. 2011, MNRAS, 416, 178

Brogan, C. L., Hunter, T. R., Cyganowski, C. J., et al. 2011, ApJL, 739, L16

Brogan, C. L., Hunter, T. R., Cyganowski, C. J., et al. 2012, in IAU Symp. 287, Cosmic Masers-from OH to Ho, ed. R. S. Booth, E. M. L. Humphreys, \& W. H. T. Vlemmings (Cambridge: Cambridge Univ. Press), 497

Caselli, P. 2011, in IAU Symp. 280, The Molecular Universe, ed. J. Cernicharo \& R. Bachiller (Cambridge: Cambridge Univ. Press), 19

Caselli, P., Benson, P. J., Myers, P. C., \& Tafalla, M. 2002, ApJ, 572, 238

Ceccarelli, C., Caselli, P., Bockelee-Morvan, D., et al. 2014, arXiv:1403.7143

Ceccarelli, C., \& Dominik, C. 2005, in Proc. of the Dusty and Molecular Universe. A Prelude to Herschel and ALMA, ed. A. Wilson (ESA SP-577, Noordwijk: ESA), 349

Cyganowski, C. J., Brogan, C. L., Hunter, T. R., \& Churchwell, E. 2009, ApJ, 702,1615

Cyganowski, C. J., Brogan, C. L., Hunter, T. R., \& Churchwell, E. 2011a, ApJ, 743, 56 
Cyganowski, C. J., Brogan, C. L., Hunter, T. R., Churchwell, E., \& Zhang, Q. 2011b, ApJ, 729, 124

Cyganowski, C. J., Brogan, C. L., Hunter, T. R., et al. 2012, ApJL, 760, L20

Cyganowski, C. J., Koda, J., Rosolowsky, E., et al. 2013, ApJ, 764, 61

Cyganowski, C. J., Whitney, B. A., Holden, E., et al. 2008, AJ, 136, 2391

Duarte-Cabral, A., Bontemps, S., Motte, F., et al. 2013, A\&A, 558, A125

Garrod, R. T., Weaver, S. L. W., \& Herbst, E. 2008, ApJ, 682, 283

Graedel, T. E., Langer, W. D., \& Frerking, M. A. 1982, ApJS, 48, 321

Hofner, P., \& Churchwell, E. 1996, A\&AS, 120, 283

Hunter, T. R., Brogan, C. L., Cyganowski, C. J., \& Young, K. H. 2014, ApJ, 788, 187

Kauffmann, J., Pillai, T., \& Goldsmith, P. F. 2013, ApJ, 779, 185

McKee, C. F., \& Tan, J. C. 2002, Natur, 416, 59

McKee, C. F., \& Tan, J. C. 2003, ApJ, 585, 850

Mocanu, L. M., Crawford, T. M., Vieira, J. D., et al. 2013, ApJ, 779, 61

Molinari, S., Pezzuto, S., Cesaroni, R., et al. 2008, A\&A, 481, 345

Myers, A. T., McKee, C. F., Cunningham, A. J., Klein, R. I., \& Krumholz, M. R. 2013, ApJ, 766, 97

Ossenkopf, V., \& Henning, T. 1994, A\&A, 291, 943

Padovani, M., Galli, D., \& Glassgold, A. E. 2009, A\&A, 501, 619

Padovani, M., Hennebelle, P., \& Galli, D. 2013, A\&A, 560, A114
Pineau des Forets, G., Flower, D. R., \& Chieze, J.-P. 1997, in IAU Symp. 182, Herbig-Haro Flows and the Birth of Stars, ed. B. Reipurth \& C. Bertout (Dordrecht: Kluwer), 199

Rathborne, J. M., Lada, C. J., Muench, A. A., et al. 2009, ApJ, 699, 742

Sato, M., Wu, Y. W., Immer, K., et al. 2014, ApJ, 793, 72

Semenov, D., Henning, T., Helling, C., Ilgner, M., \& Sedlmayr, E. 2003, A\&A, 410,611

Smith, R. J., Longmore, S., \& Bonnell, I. 2009, MNRAS, 400, 1775

Tan, J. C., Beltran, M. T., Caselli, P., et al. 2014, arXiv:1402.0919

Tan, J. C., Kong, S., Butler, M. J., Caselli, P., \& Fontani, F. 2013, ApJ, 779, 96

Tobin, J. J., Hartmann, L., Chiang, H.-F., et al. 2013, ApJ, 771, 48

van der Tak, F. F. S., Black, J. H., Schöier, F. L., Jansen, D. J., \& van Dishoeck, E. F. 2007, A\&A, 468, 627

Vasyunin, A. I., \& Herbst, E. 2013, ApJ, 769, 34

Vasyunin, A. I., Semenov, D., Henning, T., et al. 2008, ApJ, 672, 629

Vasyunin, A. I., Semenov, D. A., Wiebe, D. S., \& Henning, T. 2009, ApJ, 691, 1459

Vasyunin, A. I., Sobolev, A. M., Wiebe, D. S., \& Semenov, D. A. 2004, AstL, 30,566

Wilner, D. J., \& Welch, W. J. 1994, ApJ, 427, 898

Wilson, T. L., \& Rood, R. 1994, ARA\&A, 32, 191 